\title{
LncRNA PCED1B-AS1 is Overexpressed in Hepatocellular Carcinoma and Regulates miR- 10a/BCL6 axis to Promote Cell Proliferation
}

\author{
Haitao Ding ( $\square$ HaitaoDingClinical@163.com ) \\ Inner Mongolia People's Hospital \\ Juan He \\ Inner Mongolia People's Hospital \\ Weili Xiao \\ Inner Mongolia People's Hospital \\ Zhihong Ren \\ Inner Mongolia People's Hospital \\ Wanting Gao \\ Inner Mongolia People's Hospital
}

\section{Research}

Keywords: hepatocellular carcinoma, PCED1B-AS1, miR-10a, BCL6

Posted Date: September 24th, 2020

DOl: https://doi.org/10.21203/rs.3.rs-79374/v1

License: (c) (1) This work is licensed under a Creative Commons Attribution 4.0 International License. Read Full License

Version of Record: A version of this preprint was published at Critical Reviews in Eukaryotic Gene Expression on January 1st, 2022. See the published version at https://doi.org/10.1615/CritRevEukaryotGeneExpr.2022039954. 


\section{Abstract}

Background: PCED1B-AS1 has been characterized as an oncogene in glioma, while its role in hepatocellular carcinoma (HCC) is unknown. This study was performed to analyze the involvement of PCED1B-AS1.

Methods: Expression of PCED1B-AS1 in paired HCC and non-tumor tissues from $62 \mathrm{HCC}$ patients was determined by RT-qPCR. The correlation between PCED1B-AS1 and miR-10a or BCL6 was analyzed by linear regression. The $62 \mathrm{HCC}$ patients were followed up for 5 years to analyze the prognostic value of PCED1B-AS1 for HCC. The interactions among PCED1B-AS1, miR-10a and BCL6 were analyzed by overexpression experiments. Cell proliferation was analyzed by CCK-8 assay.

Results: PCED1B-AS1 was upregulated in HCC and predicted poor survival. Across HCC tissues, PCED1BAS1 was inversely correlated with miR-10a, and positively correlated with BCL6 mRNA. In HCC cells, PCED1B-AS1 overexpression mediated the downregulation of miR-10a and upregulation of BCL6. Moreover, PCED1B-AS1 overexpression reduced the inhibitory effects of miR-10a overexpression on BCL6 expression and cell proliferation.

Conclusions: PCED1B-AS1 is overexpressed in HCC and regulates miR-10a/BCL6 axis to promote cell proliferation.

\section{Introduction}

Liver cancer is a common malignancy that accounts for a considerable portion of new cancer cases and mortalities [1]. It is estimated that liver cancer in 2018 caused a total of 781,631 deaths, accounting for $82 \%$ of all cancer deaths, and affected a total of 841,080 new cases, which were the $4.7 \%$ of all new cases [2]. Despite the advances in the diagnosis and treatment of HCC, only $44 \% \mathrm{HCC}$ patients are diagnosed at early stage, resulting in a 5-year overall survival rate of 33\% [3]. However, distant tumor metastasis is common in liver cancer, and once distant metastasis has occurred, only less than $2 \%$ of patients can survive for 2 years [4]. Therefore, novel therapeutic and preventative approaches are still needed to improve patients' survival.

It has been well established that infections of $\mathrm{HBC}$ and $\mathrm{HCV}$, cirrhosis and alcohol abuse are the major risk factors for liver cancer [5-6]. However, having these risk factors is not sufficient for the initiation and development of liver cancer [7]. In effect, a considerable number of molecular factors have been identified in the progression of liver cancer [8]. Some of the molecular factors have been proven to be potential targets for targeted therapy, which can be performed to suppress tumor by regulating regulated gene expression $[9,10]$. However, effect targets for targeted therapy remain lack. With protein-coding capacity, IncRNAs participate in human diseases by regulating gene expression at multiple levels [11, 12], suggesting the potential role of IncRNAs as targets for targeted therapy. PCED1B-AS1 has been characterized as an oncogene in glioma $[13,14]$, while its role in liver cancer is unknown. Our preliminary deep sequencing analysis showed that PCED1B-AS1 expression altered in hepatocellular carcinoma 
(HCC), a major subtype of liver cancer, and it is inversely correlation with miR-10a, which targets BCL6 to player tumor suppressive roles [15]. This study was therefore carried out to explore the interaction between PCED1B-AS1, miR-10a and BCL6 in HCC.

\section{Methods}

\section{HCC patients and tissue collections}

This study was approved by Ethics Committee of Inner Mongolia People's Hospital. A total of 62 HCC patients (38 males and 24 females) were enrolled at the aforementioned hospital from March 2014 to May 2015. Age of these patients ranged from 44 to 69 years ( $57.1 \pm 5.6$ years). Other clinical disorders or therapy may also affect gene expression, this study excluded patients with initiated therapy or complicated with other clinical disorders. All the 62 patients were diagnosed for the first time and no recurrent cases were included. Based on their medical record, HBV-positive was observed in 23 cases, HCV-positive was observed in 29 cases, and the rest 10 cases were negative for both. Prior to therapy, $\mathrm{HCC}$ and paired non-tumor tissues were collected through fine needle aspiration from all patients. Tissues were confirmed by histopathological exam and were stored in a liquid nitrogen tank before use. Patients signed informed consent.

\section{Treatments and follow-up}

The 62 groups were classified into AJCC stage I $(n=8)$, II $(n=12)$, III $(n=20)$ and IV $(n=22)$. Therapies were determined according to their AJCC stages and other medical conditions. From the day of admission, patients were visited monthly to record their survival conditions. Patients died of caused unrelated to HCC were not included.

\section{HCC cells and transient transfections}

HCC cell line SNU-182 from ATCC (USA) was included as the cell model of HCC. RPMI 1640 medium supplemented with $10 \%$ FBS was used as cell culture medium. At $37{ }^{\circ} \mathrm{C}$, SNU-182 cells were cultivated in a $5 \% \mathrm{CO}_{2}$ and $95 \%$ humidity incubator.

Backbone expression vector of PCED1B-AS1 or BCL6 was established using pcDNA3.1 vector (Invitrogen). Mimic of miR-10a and negative control (NC) miRNA were synthesized by Sigma-Aldrich. Through lipofectamine 2000 (Invitrogen)-mediated transient transfections, SNU-182 cells were transfected with $1 \mu \mathrm{g}$ expression vector or $35 \mathrm{nM}$ miRNA. NC experiments were performed by transfecting the same amount of empty vector or NC miRNA into the same number of cells. Prior to subsequent experiments, cells were cultivated in fresh medium for $48 \mathrm{~h}$. 


\section{RNA preparations}

Total RNA isolation from SNU-182 cells and tissue samples was performed using Ribozol reagent (Invitrogen). DNase I was used to incubate RNA samples for $2 \mathrm{~h}$ at $37^{\circ} \mathrm{C}$ for complete genomic DNA removal.

\section{RT-qPCRs}

Using SS-IV-RT system (Invitrogen), RNA samples were used as template to synthesize cDNA through reverse transcriptions. QuantiFast SYBR Green PCR Kit (QIAGEN) was them used to perform qPCRs with GAPDH as internal control to determine the expression of PCED1B-AS1 and BCL6 mRNA. Mature miR-10a expression was determined using All-in-One ${ }^{\mathrm{TM}}$ miRNA qRT-PCR Reagent Kit (Genecopoeia) with all steps performed following manufacturer's instructions. U6 was used as the internal control of miR-10a. Each qPCR was performed in three technical replicates and $2^{-\Delta \Delta C q}$ was used to normalize Ct values of target genes to internal controls.

\section{Western blot}

Total protein isolation from transfected cells and protein quantification were performed using RIPA solution (Invitrogen) and BCA assay, respectively. At $95^{\circ} \mathrm{C}$, protein samples were incubated for $15 \mathrm{~min}$ for protein denaturation. A $6 \%$ SDS-PAGE gel was used to separate proteins, and PVDF membranes were used to transfer proteins. To block membranes, PBS containing $5 \%$ non-fat milk was used to incubate membranes for $2 \mathrm{~h}$ at $25^{\circ} \mathrm{C}$. Primary antibodies of BCL6 (ab203619, Abcam) and GAPDH (ab9485, Abcam) were used to incubate membranes at $4^{\circ} \mathrm{C}$ for $12 \mathrm{~h}$. After that, secodary antibody of IgG-HRP (ab6721, Abcam) was used to incubate the membranes at $25^{\circ} \mathrm{C}$ for $2 \mathrm{~h}$. ECL (Invitrogen) was dropped onto membranes to develop signals. Data normalization was performed using Quantity One software.

\section{CCK-8 kit}

SNU-182 cells with transfections were harvetsed and were subjected to CCK-8 cell proliferation analysis. Cells were transferred to a 96-well cell culture plate with 4000 cells in $0.1 \mathrm{ml}$ fresh medium. At $37^{\circ} \mathrm{C}$, cells were cultivated and CCK- 8 solution was added into each well to reach $10 \%$ at $2 \mathrm{~h}$ before the measurement of OD values. At $450 \mathrm{~nm}, \mathrm{OD}$ values were measured every $24 \mathrm{~h}$ for a total of 4 days.

\section{Statistical analysis}

Gene expression levels in paired tissues from HCC patients were expressed as average values of three technical replicates and data were compared by paired t test. Data of multiple cell transfection groups were expressed as mean $\pm S D$ values and were compared by ANOVA Tukey's test. Correlations were 
analyzed by linear regression. To analyze the prognostic value of PCED1B-AS1 for HCC, patients were grouped into high and low PCED1B-AS1 level groups $(n=31)$. Survival curves were plotted for both groups and log-rank test was used for survival curve comparison. $\mathrm{P}<0.05$ was deemed statistically significant.

\section{Results}

\section{Overexpression of PCED1B-AS1 predicted the poor survival of HCC patients.}

Expression of PCED1B-AS1 in paired HCC and non-tumor tissues from $62 \mathrm{HCC}$ patients was determined by RT-qPCR. Significantly overexpressed expression of PCED1B-AS1 was observed in HCC tissues in comparison to non-tumor tissues (Fig.1A, $p<0.001$ ). Survival curve analysis showed that, overall survival was significantly worse in high PCED1B-AS1 level group compared to low PCED1B-AS1 level group (Fig.1B). Interestingly, HBV and HCV infections failed to significantly affect the expression of PCED1BAS1 in both HCC and non-tumor tissues (data not shown).

\section{Correlations among PCED1B-AS1, miR-10a and BCL6 mRNA across HCC tissues}

Expression of miR-10a and BCL6 mRNA in HCC tissues form the $62 \mathrm{HCC}$ patients was also determined by RT-qPCR. Correlation analysis performed using linear regression showed that PCED1B-AS1 was inversely and significantly correlated with miR-10a (Fig.2A), and positively and significantly correlated with BCL6 mRNA (Fig.2B) across HCC tissues. Moreover, miR-10a and BCL6 mRNA were inversely and significantly correlated across HCC tissues (Fig.2C). These data suggest the existence of interactions among PCED1BAS1, miR-10a and BCL6 in HCC.

\section{PCED1B-AS1 overexpression increased BCL6 expression through miR-10a}

To explore the interactions among PCED1B-AS1, miR-10a and BCL6, SNU-182 cells were transfected with PCED1B-AS1 expression vector or miR-10a mimic, and the overexpression of PCED1B-AS1 and miR-10a was confirmed by RT-qPCR at 48h post-transfection (Fig.3A, p<0.05). It was observed that PCED1B-AS1 overexpression significant decreased the expression of miR-10a (Fig.3B, $p<0.05$ ), while miR-10a overexpression failed to significantly affect the expression of PCED1B-AS1 (Fig.3C). BCL6 is a validated target of miR-10a, the effects of PCED1B-AS1 and miR-10a overexpression on the expression of BCL6 mRNA and protein were analyzed by RT-qPCR and Western blot, respectively. It was observed that PCED1B-AS1 overexpression increased BCL6 expression, while miR-10a overexpression decreased BCL6 expression. Moreover, PCED1B-AS1 overexpression reduced the inhibitory effects of miR-10a overexpression on BCL6 expression (Fig.3D, p<0.05). 


\section{PCED1B-AS1 overexpression increased HCC cell proliferation through miR-10a/BCL6 axis}

The role of PCED1B-AS1, miR-10a and BCL6 in regulating the proliferation of SNU-182 cells was explored by CCK-8 assay. PCED1B-AS1 and BCL6 overexpression significantly increased cell proliferation, while miR-10a significantly decreased cell proliferation. Moreover, PCED1B-AS1 overexpression reduced the inhibitory effects of miR-10a overexpression on cell proliferation (Fig.4, p<0.05).

\section{Discussion}

This study explored the interactions between PCED1B-AS1, miR-10a and BCL6 in HCC. We found that PCED1B-AS1 was overexpressed in HCC and was correlated with the survival of HCC patients. In addition, PCED1B-AS1 could downregulate miR-10a to upregulate BCL6, thereby promoting the proliferation of HCC cells.

Previous studies have explored the involvement of PCED1B-AS1 in glioma [13, 14]. It was reported that PCED1B-AS1 was overexpressed in glioma and regulates miR-194-5p/PCED1B to increase cell apoptosis [13]. In another study, PCED1B-AS1 was reported to upregulate HIF-1a in glioblastoma, which in turn promotes Warburg effect and tumorigenesis [14]. However, the role of PCED1B-AS1 in other cancers is unknown. In this study we observed the upregulation of PCED1B-AS1 in HCC and PCED1B-AS1 overexpression increased HCC cell proliferation. Therefore, PCED1B-AS1 plays oncogenic roles in HCC by promoting cancer cell proliferation. HBV and HCV infections contribute to the development of HCC [16]. In this study, no significant differences in expression levels of PCED1B-AS1 were observe among different $\mathrm{HBV}$ and HCV infection groups. Therefore, PCED1B-AS1 may participate in HCC through pathways independent from HBV and HCV infections.

Despite the advances in HCC treatment and diagnosis, the overall survival of HCC patients is still poor [3, 4]. In this study we showed that the high expression levels of PCED1B-AS1 were closely correlated with the poor survival of HCC patients, suggesting that PCED1B-AS1 may serve as a prognostic factor for HCC. However, more clinical trials are needed to further analyze the reliability of the using of PCED1B-AS1 as a prognostic factor for HCC.

MiR-10a plays different roles in different cancers $[15,17]$. MiR-10a is downregulated in large B-cell lymphoma and promotes cell apoptosis and suppresses cell proliferation to play tumor suppressive roles [15]. In contrast, miR-10a is overexpressed in cervical cancer and targets CHL1 to promote cancer cell invasion, migration and proliferation, suggesting its oncogenic roles [17]. In this study we showed that miR-10a could target BCL6 to suppress HCC cell proliferation. The key finding is that PCED1B-AS1 overexpression decreased the expression of miR-10a to upregulate BCL6. However, the mechanism remains unclear. More studies are still needed to explore the molecular mechanism.

\section{Conclusion}


In conclusion, PCED1B-AS1 is overexpressed in HCC and predicts poor survival of HCC patients. In addition, PCED1B-AS1 may downregulate miR-10a to upregulate BCL6, thereby promoting HCC cell proliferation.

\section{Declarations}

\section{Ethics approval and consent to participate}

The study was granted ethical approval by the Ethical Committee of Inner Mongolia People's Hospital, and all the patients or parents/ guardians of patients provided written informed consent.

\section{Consent for publication}

Not applicable.

\section{Availability of data and material}

The datasets used and/or analyzed during the current study are available from the corresponding authors upon reasonable request.

\section{Competing interests}

The authors declare that they have no competing interests.

\section{Funding}

This work was supported by The Inner Mongolia Science and Technology Application Pproject (Grant No. 201602097).

\section{Authors' contributions}

Haitao Ding designed the study. Haitao Ding carried out experiments and wrote the manuscipt, Haitao Ding revised the paper, Juan He, Weili Xiao, Zhihong Ren and Wanting Gao collected patient specimens and related information. Juan He, Weili Xiao, Zhihong Ren, Wanting Gao contributed to analysing the data. All authors reviewed the results and approved the final version of the manuscript.

\section{Acknowledgements}


The authors would like to thank the patients who participated in this survey.

\section{References}

1. Siegel RL, Miller KD, Jemal A. Cancer statistics, 2019. CA Cancer J Clin. 2019;69(1):7-34.

2. Bray F, Ferlay J, Soerjomataram I, et al. Global cancer statistics 2018: GLOBOCAN estimates of incidence and mortality worldwide for 36 cancers in 185 countries. CA Cancer J Clin. 2018;68(6):394-424.

3. He XK, Lin ZH, Qian Y, et al. Marital status and survival in patients with primary liver cancer. Oncotarget. 2017;8(39):64954-63.

4. Wong MCS, Jiang JY, Goggins WB, et al. International incidence and mortality trends of liver cancer: a global profile. Sci Rep. 2017;7:45846.

5. Wang $M$, Wang $Y$, Feng $X$, et al. Contribution of hepatitis $B$ virus and hepatitis $C$ virus to liver cancer in China north areas: Experience of the Chinese National Cancer Center. Int J Infect Dis. 2017;65:15-21.

6. Stoian M, Stoica V. Risk Factors for Hepatocellular Carcinoma. Intern Med. 2018;15(6):7-20.

7. Ho DWH, Lo RCL, Chan LK, et al. Molecular pathogenesis of hepatocellular carcinoma. Liver Cancer. 2016;5(4):290-302.

8. Sia D, Villanueva A, Friedman SL, et al. Liver cancer cell of origin, molecular class, and effects on patient prognosis. Gastroenterology. 2017;152(4):745-61.

9. Chen S, Cao Q, Wen W, et al. Targeted therapy for hepatocellular carcinoma: Challenges and opportunities. Cancer Lett. 2019;460:1-9.

10. Llovet JM, Montal R, Sia D, et al. Molecular therapies and precision medicine for hepatocellular carcinoma. Nat Rev Clin Oncol. 2018;15(10):599-616.

11. Prensner JR, Chinnaiyan AM. The emergence of IncRNAs in cancer biology. Cancer Discov. 2011;1(5):391-407.

12. Huarte M. The emerging role of IncRNAs in cancer. Nat Med. 2015;21(11):1253.

13. Yang J, Yu D, Liu X, et al. LncRNA PCED1B-AS1 activates the proliferation and restricts the apoptosis of glioma through cooperating with miR-194-5p/PCED1B axis. J Cell Biochem. 2020;121(2):182333.

14. Yao Z, Zhang Q, Guo F, et al. Long Noncoding RNA PCED1B-AS1 Promotes the Warburg Effect and Tumorigenesis by Upregulating HIF-1 a in Glioblastoma. Cell Transplant. 2020;29:963689720906777.

15. Fan Q, Meng X, Liang H, et al. miR-10a inhibits cell proliferation and promotes cell apoptosis by targeting BCL6 in diffuse large B-cell lymphoma. Protein Cell. 2016;7(12):899-912.

16. Rongrui $\mathrm{L}, \mathrm{Na} \mathrm{H}$, Zongfang L, et al. Epigenetic mechanism involved in the HBV/HCV-related hepatocellular carcinoma tumorigenesis. Curr Pharm Des. 2014;20(11):1715-25.

17. Long MJ, Wu FX, Li P, et al. MicroRNA-10a targets CHL1 and promotes cell growth, migration and invasion in human cervical cancer cells. Cancer Lett. 2012;324(2):186-96. 
Figures

A

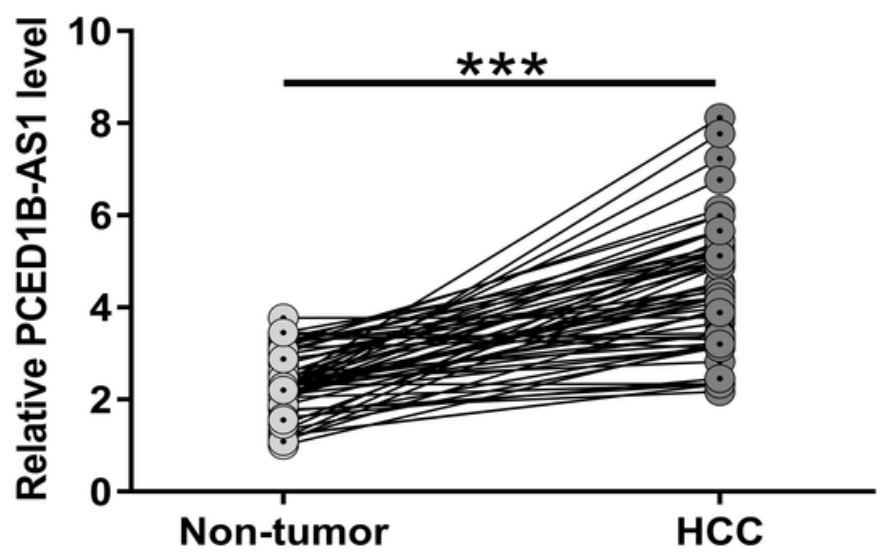

B

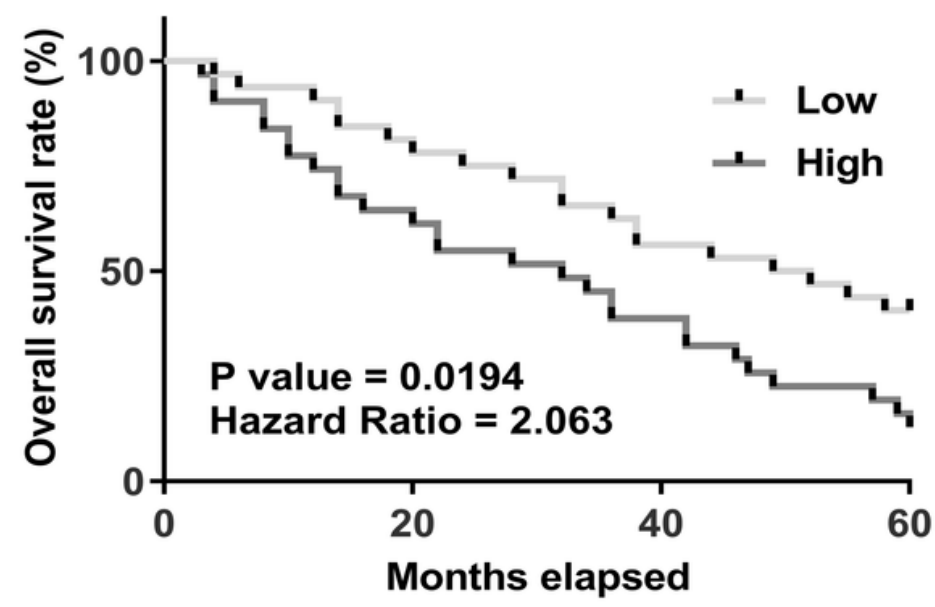

Figure 1

Overexpression of PCED1B-AS1 predicted the poor survival of HCC patients. Expression of PCED1B-AS1 in paired HCC and non-tumor tissues from $62 \mathrm{HCC}$ patients was determined by RT-qPCR. Gene expression levels in paired tissues from HCC patients were expressed as average values of three technical replicates. ${ }^{\star \star \star}, p<0.001$. To analyze the prognostic value of PCED1B-AS1 for HCC, patients were grouped into high and low PCED1B-AS1 level groups $(n=31)$. Survival curves were plotted for both groups and logrank test was used for survival curve comparison (B). 

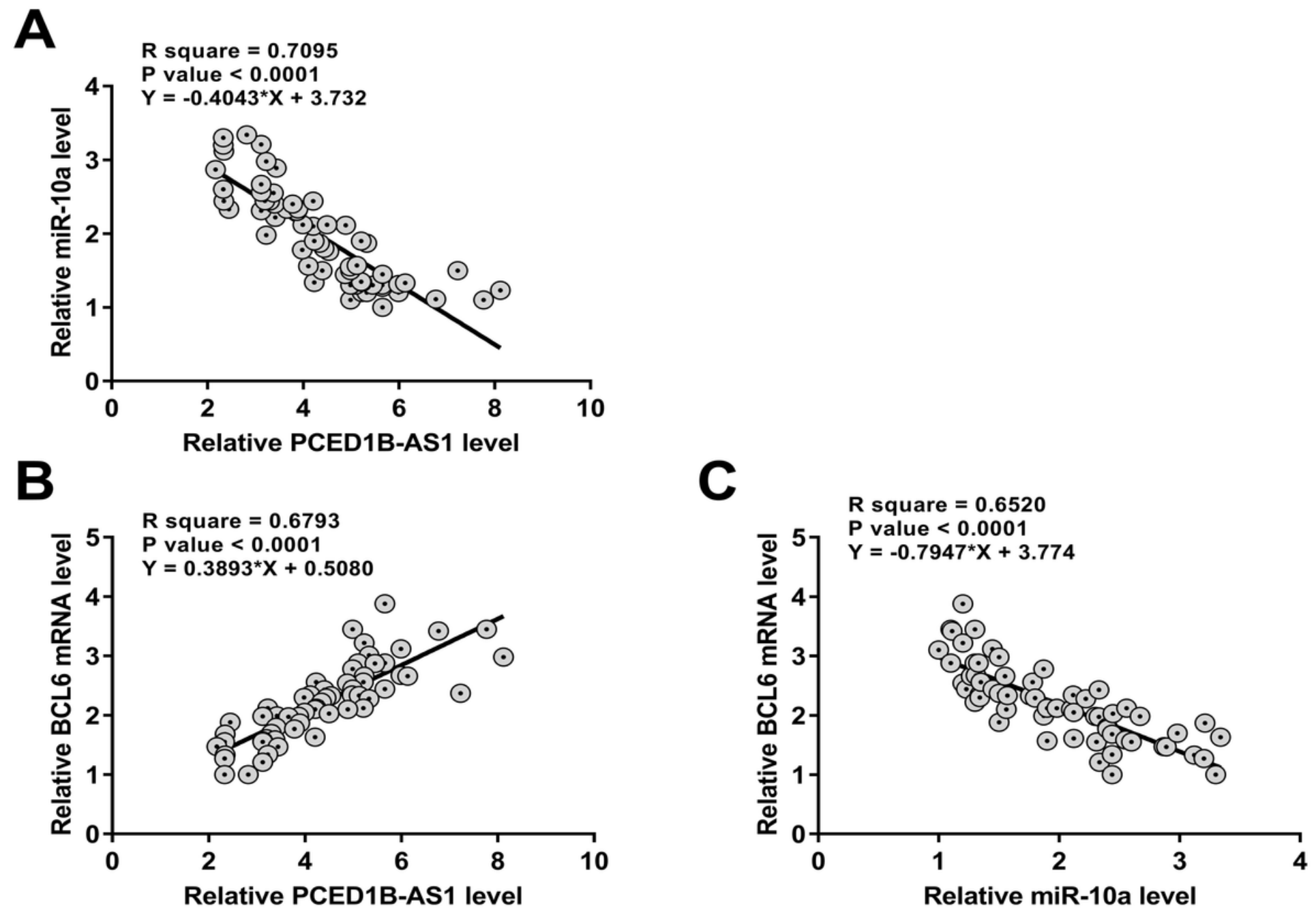

Figure 2

Correlations among PCED1B-AS1, miR-10a and BCL6 mRNA across HCC tissues Expression of miR-10a and BCL6 mRNA in HCC tissues form the $62 \mathrm{HCC}$ patients was also determined by RT-qPCR. Correlations between PCED1B-AS1 and miR-10a (A), between PCED1B-AS1 and BCL6 mRNA (B), and between miR$10 a$ and BCL6 mRNA (C) across HCC tissues were analyzed by linear regression. 

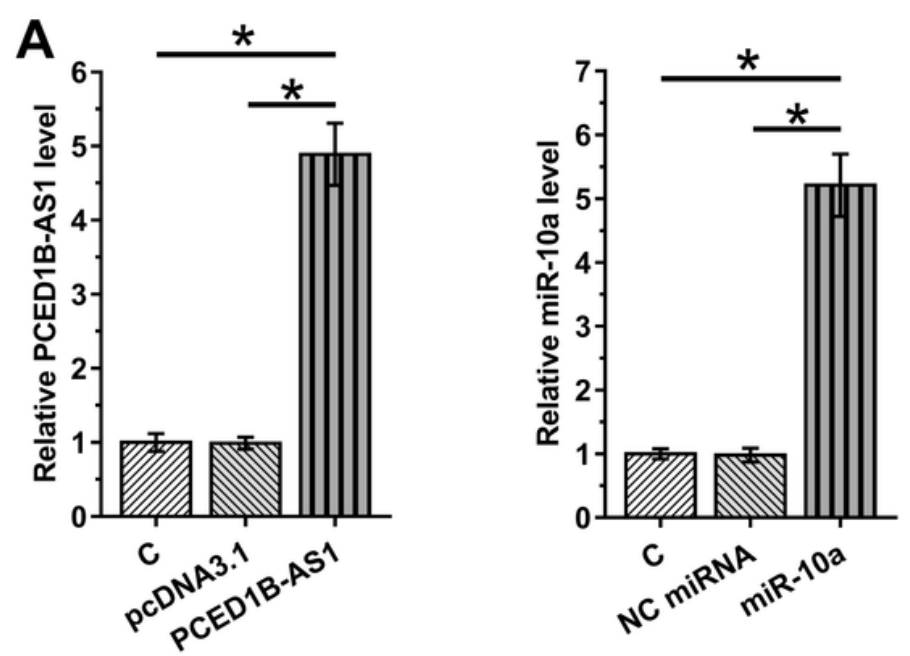

B

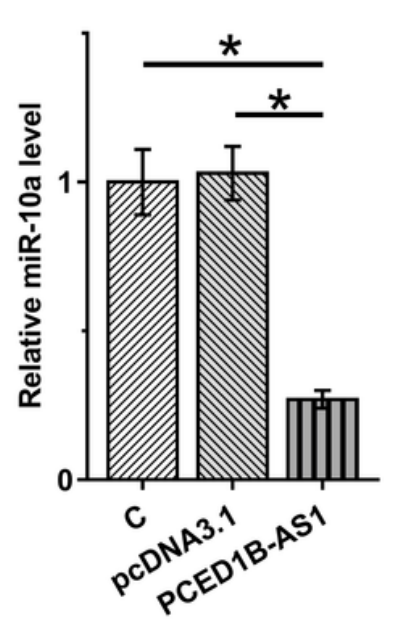

C

D
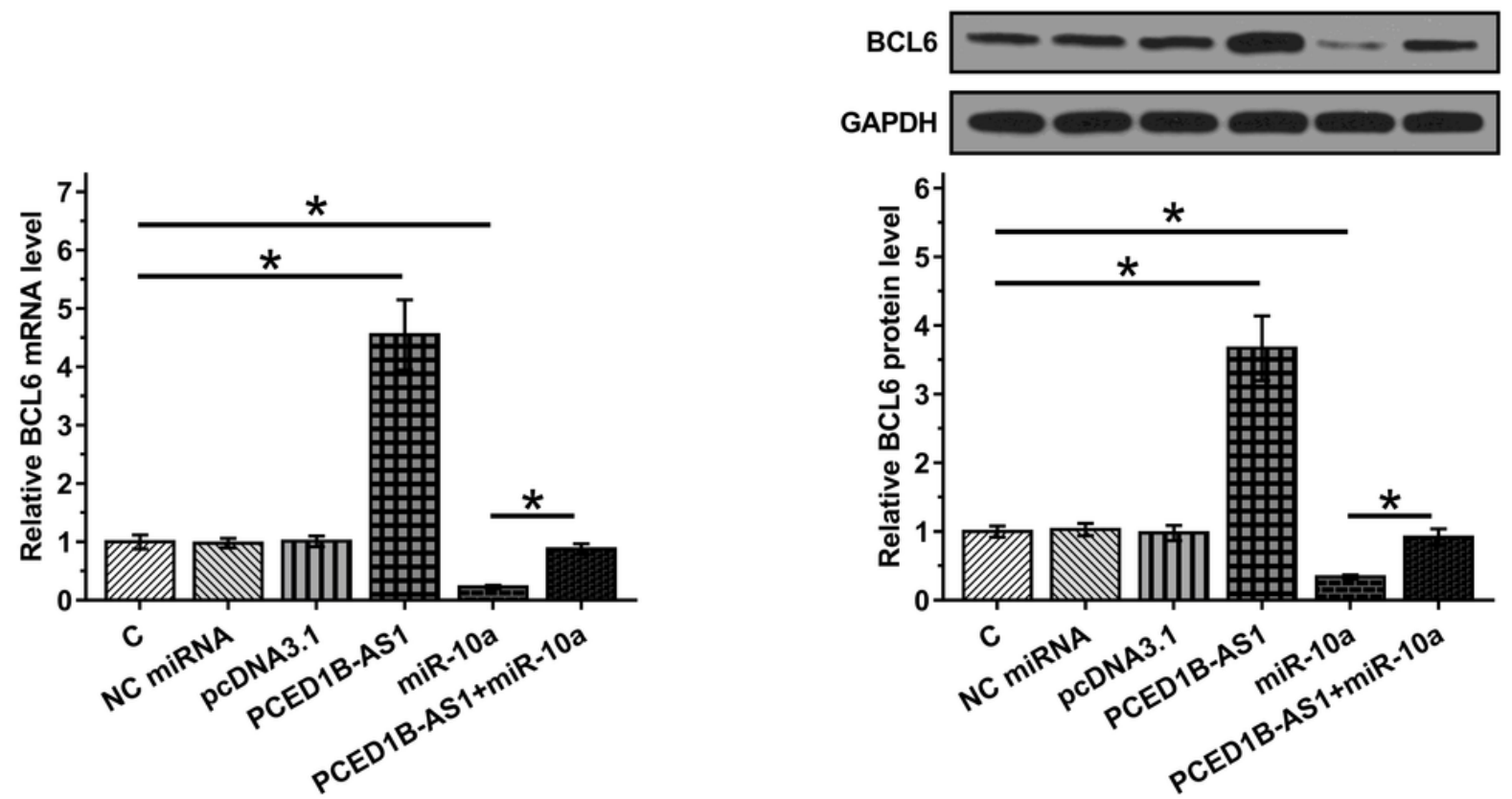

Figure 3

PCED1B-AS1 overexpression increased BCL6 expression through miR-10a To explore the interactions among PCED1B-AS1, miR-10a and BCL6, SNU-182 cells were transfected with PCED1B-AS1 expression vector or miR-10a mimic, and the overexpression of PCED1B-AS1 and miR-10a was confirmed by RTqPCR at 48h post-transfection (A). The effects of PCED1B-AS1 overexpression on miR-10a (B) and the effects of miR-10a overexpression on PCED1B-AS1 (C) were explored by RT-qPCR. The effects of PCED1B-AS1 and miR-10a overexpression on the expression of BCL6 mRNA and protein were analyzed by RT-qPCR and Western blot, respectively (D). Data of multiple cell transfection groups were expressed as mean $\pm S D$ values. ${ }^{*}, p<0.05$. 


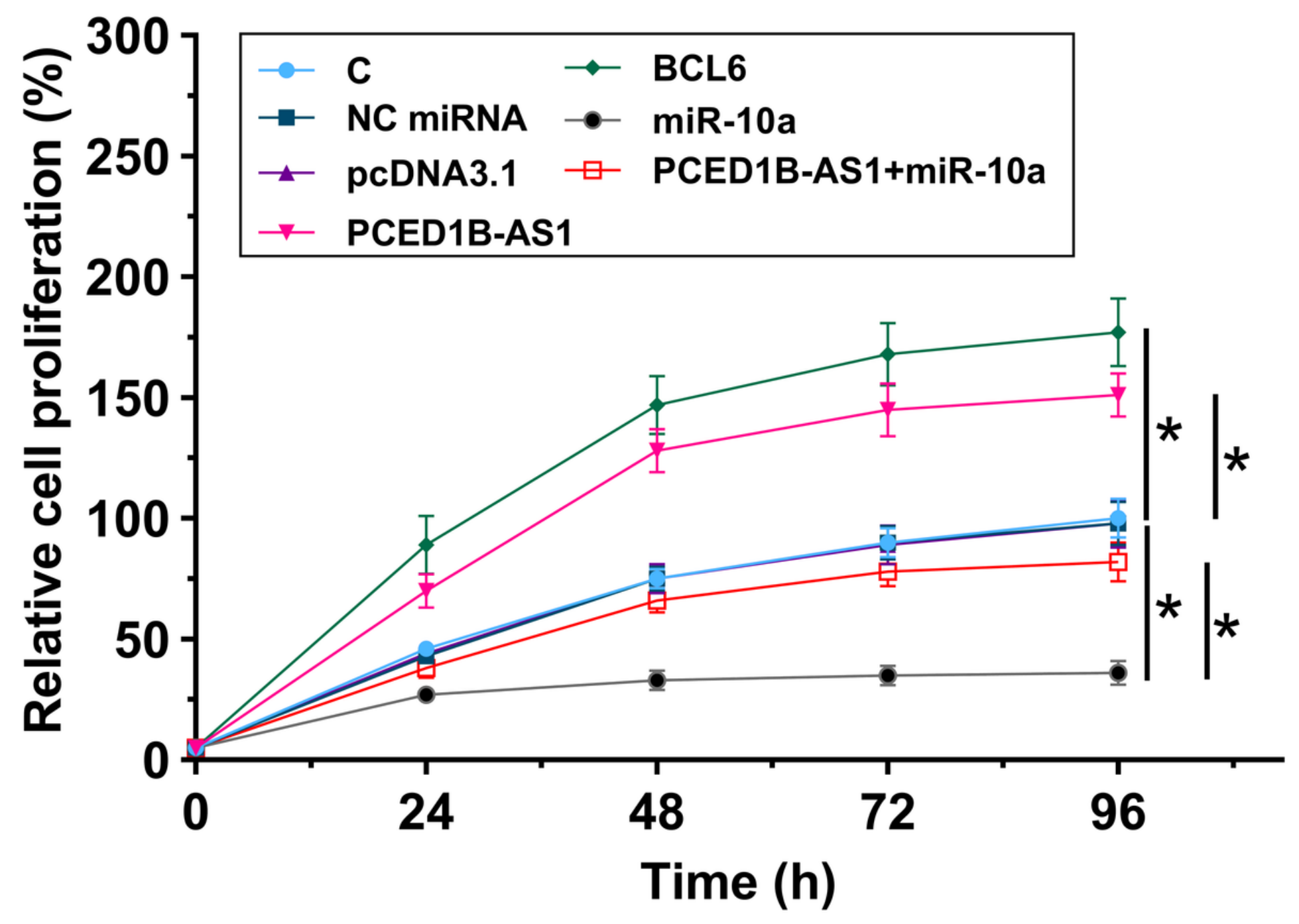

Figure 4

PCED1B-AS1 overexpression increased HCC cell proliferation through miR-10a/BCL6 axis The role of PCED1B-AS1, miR-10a and BCL6 in regulating the proliferation of SNU-182 cells was explored by CCK-8 assay. Data of multiple cell transfection groups were expressed as mean $\pm S D$ values. ${ }^{*}, p<0.05$. 